\title{
Correction to: Multilayer Partially Homomorphic Encryption Text Steganography (MLPHE-TS): A Zero Steganography Approach
}

\author{
Nuzhat Naqvi ${ }^{1}$ - Aliya Tabassum Abbasi ${ }^{3} \cdot$ Rasheed Hussain $^{2}$ (D) $\cdot$ M. Aihab Khan ${ }^{3}$. \\ Basheer Ahmad ${ }^{3}$
}

Published online: 13 March 2019

(c) Springer Science+Business Media, LLC, part of Springer Nature 2019

\section{Correction to: Wireless Pers Commun (2018) 103:1563-1585 https://doi.org/10.1007/s11277-018-5868-1}

The full name of the first author's affiliation was not shown in the original publication. It is correctly shown here.

The original article can be found online at https://doi.org/10.1007/s11277-018-5868-1.

Rasheed Hussain

r.hussain@innopolis.ru

Nuzhat Naqvi

nuzhatnaqvi@mail.ustc.edu.cn

Aliya Tabassum Abbasi

Aliyatabassumabbasi@gmail.com

M. Aihab Khan

aihab@iqraisb.edu.pk

Basheer Ahmad

drbasheer@iqraisb.edu.pk

1 University of Science and Technology of China (USTC), Hefei, China

2 Innopolis University, Innopolis, Russia

3 Iqra University, Islamabad, Pakistan 